\title{
TESTE DE BENDER (B-SPG) E DFH-ESCALA SISTO: VALIDADE POR GRUPOS CONTRASTANTES
}

\section{Daniel Bartholomeu}

Psicólogo, mestre e doutor em avaliação psicológica pela Universidade São Francisco. Docente na Faculdade Anhanguera de Jundiaí e no Centro Universitário Salesiano de Americana onde coordena o Laboratório de Psicodiagnóstico e Neurociências Cognitivas (LAPENC).

Juliana Francisca Cecato Bióloga, discente do curso de Psicologia - Anhanguera Educacional, mestranda do Programa de Pós Graduação - Faculdade de Medicina de Jundiaí.

José Maria Montiel

Psicólogo, especialista em Diagnóstico e Triagem, Mestre e Doutor em Avaliação Psicológica em Contextos de Saúde Mental - Universidade São Francisco. Programa de Pós-Graduação e membro do Núcleo de Pesquisa do Instituto de Pesquisas Aplicadas e Desenvolvimento Educacional (IPADE)

- Anhanguera Educacional. Pesquisador colaborador do Laboratório de Psicodiagnóstico e Neurociências Cognitivas (LAPENC) UNISAL.

\section{Afonso Antonio Machado}

Formado pela PUC em Educação Física, mestrado e doutorado em Educação pela Universidade Estadual de Campinas. Livre docente pela Universidade Estadual Paulista Júlio de Mesquita Filho. Colaborador do Núcleo de Estudos e Pesquisa em Psicologia do Esporte (NEPESPE). Professor adjunto da Universidade Estadual Paulista Júlio de Mesquita Neto (UNESP - Rio Claro).

Fermino Fernandes Sisto Doutor pela Universidad Complutense de Madrid, livre docente pela Unicamp e docente do Programa de Pós Graduação Strictu Sensu da Universidade São Francisco. Coordenador do Laboratório de Avaliação Psicológica e Educacional.

\section{Resumo}

Este trabalho investigou evidências de validade por grupos contrastantes para o Teste de Bender (B-SPG) e o DFH-Escala Sisto. Os instrumentos foram aplicados a 244 alunos, 50,4\% meninos, com idades de sete a 10 anos, média de oito anos $(D P=1,08)$ que cursavam de primeira a quarta série do ensino fundamental de uma escola pública do interior do estado de São Paulo. Os testes foram aplicados coletivamente, sendo que no caso do Bender as figuras foram projetadas no quadro negro para que pudessem ser copiadas pelas crianças. Foram evidenciadas diferenças entre os grupos extremos do DFH em relação ao escore do teste de Bender em todas as situações estudadas. Concluiu-se que o Teste Gestáltico fornece uma estimativa das capacidades intelectuais de crianças e diferencia aquelas com maiores pontuações no DFH das com menores pontuações nesse teste.

Palavras-chave: avaliação psicológica; teste de Bender; desenho da figura humana; psicodiagnóstico; desenvolvimento humano. 


\title{
BENDER TEST (B-SPG) AND HFD-ESCALA SISTO: VALIDITY BY CONTRASTING GROUPS
}

\begin{abstract}
This study investigated validity evidences by contrasting groups for the Bender (BSPG) and DFH-Escala Sisto. The instruments were administered to 244 pupils, being $50.4 \%$ boys and all of them aged from seven to 10 years, with an average of eight years $(D P=1,08)$ attending from first to fourth grades of the basic education of a public school in the state of São Paulo. The tests were collectively administered and the slides of the Bender test were projected in the blackboard to be copied by the children. Differences between the extreme groups of the DFH in relation to the Bender scores were evidenced in all the studied situations. It was concluded that the Gestaltic Test furnishes an estimative of the intellectual capacities of children and differentiates those with bigger scores in the DFH from those less scored.
\end{abstract}

Keywords: psychological assessment; Bender test; human figure drawing; psychodiagnostic; human development.

\section{TEST DE BENDER (B-SPG) E DFH-ESCALA SISTO: VALIDEZ POR GRUPOS DE CONTRASTES}

\begin{abstract}
Resumen
Este estudio investigó la evidencia de validez por grupos contrastantes para la prueba de Bender (B-SPG) y DFH Escala-Sisto. Los instrumentos fueron administrados a 244 estudiantes, $50,4 \%$ hombres, con edades entre siete y 10 años, promedio de ocho años $(D E=1,08)$ asistiendo desde el primero a cuarto grado en una escuela pública en el estado de São Paulo. Las pruebas fueron aplicadas colectivamente, y en el caso de las figuras del test de Bender fueron proyectadas en una pizarra de manera que pudiera ser copiada por los niños. No hubo diferencias entre los grupos en cuanto a los extremos del puntaje de la prueba DFH obtenido en todos los casos estudiados. Llegamos a la conclusión de que el test de Bender ofrece una estimación de las capacidades intelectuales de los niños y diferencia los con puntajes más altos y bajos en el DFH.
\end{abstract}

Palabras clave: evaluación psicológica; Bender; dibujo de figura humana; psicodiagnóstico; desarrollo humano.

\section{INTRODUÇÃO}

Todo teste psicológico deve apresentar certas características que lhe garantam avaliações mais adequadas dos fenômenos a serem medidos. Tais aspectos asseguram que os resultados fornecidos pelos instrumentos sejam mais confiáveis. Dentre essas condições encontram-se a padronização, precisão e validade (Bartholomeu, Rueda \& Sisto, 2006). Segundo Anastasi e Urbina (2000) a validade informa se o teste mensura, de fato, o que se propõe a medir. Indo além dessa definição, ela nos informa sobre as inúmeras possibilidades de interpretação dos escores de um teste, e o quanto o suporte teórico está representado no mesmo. Para isso, é necessário o acúmulo de evidências que se complementem no sentido de fornecer uma compreensão mais ampla de seus 
escores (Sisto, Santos \& Noronha, 2006). Nesse contexto, pode-se dizer que não se valida um teste, mas interpretação dos dados obtidos por ele, sendo que a validade deve ser estabelecida para cada uso específico de um dado instrumento. Para Cronbach (1996), a validação é um processo de coleta de informações que dá suporte para as inferências que serão feitas com base nas pontuações do teste. Vale ressaltar que a escolha por um tipo de estudo ou outro deve ser feita em razão da finalidade do teste. Assim, a evidência pode ser circunscrita somente a uma decisão ou situação e talvez não o seja para outras.

$\mathrm{Na}$ validade concorrente, as medidas do instrumento e do critério são tomadas de forma simultânea. Assim, são retiradas duas medidas de um mesmo sujeito por meios diferentes. Uma delas é do teste que se pretende validar. A outra pode ser uma medida direta do critério, ou de outro instrumento. Tais medidas são, de forma geral, correlacionadas, para se observar se, de fato, os instrumentos convergem em suas avaliações. Outra forma de se obter essa validade seria separando grupos extremos na medida do critério e verificando se são diferenciados em razão das pontuações do instrumento que se quer validar (Anastasi \& Urbina, 2000; Bartholomeu, Rueda \& Sisto, 200). Dentro desse contexto, considerando a necessidade de que todo instrumento apresente evidências de sua validade, e que devam ser acumuladas para se ampliar a compreensão dos escores dos testes, este estudo se insere em busca do aprimoramento dessa qualidade, procurando fornecer mais evidências de validade no caso específico do Teste Gestáltico Visomotor de Bender. Esse teste foi construído tendo por base os aportes teóricos da Gestalt, mais especificamente os estudos de Max Wertheimer sobre as leis de organização perceptual. Partiu-se da premissa de que a percepção e reprodução das figuras eram determinadas por princípios biológicos e de ação sensório-motriz, que variariam de acordo com o desenvolvimento e seu estado patológico (Bender, 1938; Sisto, Noronha \& Santos, 2006).

Desde então o Teste de Bender têm sido amplamente empregado na clínica psicológica. Sundberg (1961) já ressaltava que esse instrumento, avaliado pelo sistema Koppitz, constava da lista dos mais utilizados nos Estados Unidos. Ao lado disso, muitos estudos de validade têm sido feitos para esse instrumento em uma diversidade de grupos de pessoas como pacientes psiquiátricos, crianças deficientes, e com problemas de aprendizagem (Billingslea, 1948; Gobetz, 1953; Baldwin, 1950; Harriman \& Harriman, 1950). A literatura sobre o teste de 
Bender tem demonstrado, ainda, evidências de sua validade que indicam para uma relação entre seus escores e os de inteligência, sendo interpretado que a reprodução das figuras do Bender refletiria o nível intelectual de crianças. Dentre essas pesquisas, Koppitz (1989), com crianças de primeira a quarta séries, encontrou correlações negativas entre os escores do Bender com os do WISC e da Escala de Inteligência de Stanford-Binet forma $L$ em todos os níveis de idade. Vale ressaltar que no Bender os erros são pontuados, justificando a interpretação dos resultados baseados nas correlações negativas obtidas. Desta forma, concluiu que se poderia utilizar o Bender para se estimar a inteligência de forma breve.

Noutro estudo, Baer e Gale (1967) estudaram diferenças e associações entre os escores do Bender (sistema Koppitz) e o nível de inteligência com o Teste de Maturidade Mental Califórnia em crianças institucionalizadas e não institucionalizadas em uma clínica de cuidado a pacientes com atraso intelectual, de primeira série. Nos resultados evidenciou-se que crianças institucionalizadas apresentaram menores pontuações no Bender em comparação com as demais. No entanto, a diferença do Teste Gestáltico entre os grupos desapareceu ao serem retiradas as medidas de inteligência numa análise de covariância. Foram identificadas ainda correlações entre as pontuações fornecidas pelos instrumentos para o grupo de crianças institucionalizadas e não para as outras. Esses dados conduziram os autores a concluírem que o Teste Gestáltico seria uma medida de maturidade intelectual para crianças institucionalizadas. Ao lado disso, Doubros e Mascarenhas (1969), estudando as relações entre a Escala Wechsler e o Bender, verificaram correlações entre esses instrumentos variando de baixos a moderados. Também Silberberg e Feldt (1968) procuraram estabelecer configurações psicométricas no WISC e Bender para crianças de primeiras séries com problemas de leitura. A análise dos dados levou os autores a concluírem que o escore do WISC, bem como do Teste Gestáltico de Bender, mensurado pelo sistema de Koppitz, não acrescentaram informações relevantes na explicação da performance de crianças com atrasos de leitura.

Estudando o desempenho de crianças no Bender, Minnesota Perceptual Diagnostic Test (MPDT-R) e WRAT quanto ao caráter preditivo desses instrumentos em relação ao WISC-R, Vance, Fuller e Lester (1986), evidenciaram uma superioridade do MPDT-R e do WRAT em relação ao Bender na predição do QI tal como fornecido pelo WISC-R. No estudo de McNamara, Porterfield e Miller 
(1969), os autores analisaram as relações entre a Escala de Inteligência Primária Pré-Escolar de Wechsler (WPPSI), o teste de Matrizes Progressivas Coloridas de Raven e o Teste de Bender. Os autores observaram que os escores do teste Gestáltico esteve mais associado a áreas de performance do WPPSI, e seria mais apropriado para se obter uma idéia do QI de pré-escolares do que o Matrizes Progressivas Coloridas de Raven. Apresentando dados normativos para o Teste de Bender (sistema Koppitz) em crianças iranianas de primeira a quinta séries, Yousefi, Shahim, Razavieh e Mehryar (1992) apresentaram dados de precisão e validade para esse teste. Os autores encontraram, dentre os resultados, uma correlação entre o Bender e o DFH (avaliado pelo método de Goodenough-Harris) e sugeriram que o Bender poderia ser empregado para se obter uma ideia do desenvolvimento cognitivo-intelectual das crianças iranianas.

Analisando o DFH em razão das dimensões grafomotoras dos desenhos, Hilgert e Adams (1989) utilizaram o Bender pelo sistema de Koppitz para tentar predizer esses aspectos no DFH em estudantes classificados como seriamente perturbados emocionalmente e com problemas de comportamento. As medidas obtidas nos instrumentos relacionaram-se à dimensão, altura e ponto médio de localização. Essas medidas foram então correlacionadas sendo encontradas associações entre: a dimensão das figuras 2 e 8 e as dimensões dos desenhos de figura humana; a altura das figuras A, 5 e 7 com a altura dos desenhos; e entre os pontos de localização da figura 1 do Bender com a mesma medida dos DFH. Por fim, resta destacar o trabalho de Rueda, Bartholomeu e Sisto (2004) com crianças do ensino fundamental que pesquisaram as associações entre o Bender pelos critérios de Koppitz e o DFH de Goodenough. O resultado obtido indicou uma correlação negativa e significativa entre a pontuação do DFH e as medidas de distorção, integração geral, e total do Bender. Assim, uma melhor maturidade visomotora resultaria em melhor qualidade dos desenhos, sugerindo elementos comuns entre os constructos mensurados nos testes. Pode-se afirmar que o escore do Bender associou-se com a inteligência conforme medida pelo DFH.

Pode-se perceber que os estudos estrangeiros têm demonstrado associações das medidas do Bender com inteligência. Entretanto, no Brasil, o sistema Koppitz possui inúmeras restrições quanto a seu uso adequado conforme demonstrado nos trabalhos de Pinelli Jr (1990) que apontou para problemas quanto a dimensionalidade desse teste; Sisto, Santos e Noronha (2006), Sisto, Noronha e Santos (2004) e Kroeff (1988; 1992) que encontraram problemas 
quanto a validade desenvolvimental; e Bartholomeu, Rueda e Sisto (2006) que, em sua pesquisa, verificaram a validade em relação às dificuldades de aprendizagem na escrita. Preocupados com o fato de o sistema Koppitz não ter fornecido bons resultados na diferenciação das medidas por idade ou mesmo na associação com outras medidas, Sisto e colaboradores (2005) desenvolveram um novo método de pontuação para os desenhos do Bender em crianças. O Sistema de Pontuação Gradual (B-SPG) avalia somente a distorção das figuras, atribuindo pontuações em razão da magnitude dos erros. Nesse aspecto, esses critérios diferem dos do sistema Koppitz (1989) já que o último atribui pontos para as crianças em relação a quatro quesitos, rotação, perseveração, distorção de forma e integração e apenas quando a distorção é grande. O manual do B-SPG apresenta um estudo da unidimensionalidade da escala, sugerindo que os indicadores da maturidade visomotora mensuram uma única dimensão.

Ao lado disso, todas as medidas diferenciaram as idades de sete a 10 anos, conforme demonstrado pela prova de Tukey. Foi averiguado, ainda, o funcionamento diferencial desses itens por sexo, encontrando somente duas medidas favorecendo um ou outro desses grupos. Finalmente, foi feita uma hierarquização dos níveis de dificuldade dos itens, o que possibilitou outra informação na interpretação dos dados obtidos. À parte dessas evidências de validade, Suehiro (2005) investigou as relações do B-SPG com as dificuldades de aprendizagem na escrita tendo se amparado, para isso, no ADAPE, em crianças de segunda e terceira séries do Ensino Fundamental de escolas públicas e particulares. Foram observadas evidências de validade para o B-SPG tanto por grupos separados por intensidade de dificuldades de aprendizagem, como por escolaridade. Além disso, foram verificadas diferenças significativas entre as pontuações total do ADAPE e B-SPG, nas variáveis idade, e natureza da escola, exceto para o gênero. Na pesquisa de Sisto, Bartholomeu, Rueda, Santos e Noronha (2008), foram investigadas as relações entre o B-SPG e as Matrizes Progressivas Coloridas de Raven. As correlações negativas e significativas encontradas entre as medidas dos instrumentos sugeriram que, quanto mais distorções as crianças apresentaram nas cópias, menor foi a pontuação em inteligência. A análise de grupos extremos formados em razão das Matrizes Progressivas corroborou esse dado. Todavia, Sisto, Rueda e Bartholomeu (2006) estudaram a dimensionalidade do Matrizes Progressivas Coloridas de Raven, evidenciando que esse instrumento apresentou mais de uma dimensão, 
podendo-se questionar seu uso como um critério de inteligência geral, já que se propõe a mensurar o fator $\mathrm{g}$ de Spearman.

De modo geral, considerando que a literatura demonstra sistematicamente relações entre medidas de inteligência e o Bender, e que ambos os instrumentos teriam aspectos comuns entre si, como o fato de envolverem aspectos motores e de mensurarem variáveis que apresentam um caráter desenvolvimental, essa pesquisa se propôs a buscar evidências de validade para o B-SPG em relação a inteligência, tal como mensurada pelo DFH-Escala Sisto (Sisto, 2005). A partir desses aspectos, neste trabalho serão investigadas evidências de validade para o Teste de Bender em relação à inteligência tal como avaliada pelo DFH-Escala Sisto. Mais especificamente, serão separados grupos extremos com base nas pontuações do $\mathrm{DFH}$, ou seja, crianças com alta e baixa maturidade intelectual e serão comparadas em razão dos escores do B-SPG.

\section{MÉTODO}

\section{Participantes}

A amostra total foi composta por 112 estudantes que cursavam de primeira a quarta série do ensino fundamental de escolas públicas de uma cidade do interior do estado de São Paulo. Em relação ao sexo, 50,4\% eram meninos. A idade dos alunos variou de sete a 10 anos com uma média de oito anos $(D P=1,08)$. Não houve seleção de alunos e participaram somente aqueles cujos pais autorizaram. O número de crianças com maiores e menores pontuações no DFH foi 51 e 61 respectivamente, sendo que, no primeiro caso, as crianças tinham média de idade de nove anos ( $D P=0,61)$ e $51 \%$ eram meninos e sua escolaridade foi de segunda a quarta série. Já no grupo que obteve escores mais baixos no $D F H$, a média de idade foi de sete anos ( $D P=0,87$ ) e 48,5\% eram meninos com escolaridade de primeira a terceira série.

\section{Instrumentos}

Teste Gestáltico Visomotor de Bender - Sistema de Pontuação Gradual (B-SPG)

Este instrumento consiste de nove figuras $(A, 1,2,3,4,5,6,7$ e 8) que são apresentadas uma a uma, para serem copiadas pelo participante em uma folha em branco, sem auxílio mecânico. O sistema de correção e avaliação dos aspectos maturacionais desenvolvido por Sisto, Noronha e Santos (2005), 
Sistema de Pontuação Gradual (B-SPG) ressalta que se deve atribuir a cada item uma pontuação gradual. Essa variou de zero a três pontos conforme a gravidade do erro. Os erros foram avaliados em razão da presença de desvios nas figuras. Quando não houve desvios relativos à forma, atribuiu-se a pontuação zero. As Figuras $A, 1,2,3,4,5,7$ e 8 apresentam pontuações que variam de zero a dois pontos, e a Figura 6 é avaliada de zero a três pontos. A Figura 7 apresenta dois desenhos que são avaliados separadamente pelos mesmos critérios. Desse modo, a pontuação total que uma criança pode obter nesse instrumento é de 21 pontos.

Teste do Desenho da Figura Humana - DFH-Escala Sisto

Nesse teste, foi solicitado às crianças que desenhassem uma pessoa humana com o máximo de detalhes no verso da folha que fizeram a cópia das figuras do Teste Gestáltico. O tempo para a execução do desenho foi livre, bem como o uso de borracha. Nos desenhos foram observados os seguintes indicadores, de acordo com os critérios propostos por Sisto (2005): boca, nariz, braços-perna, roupa, pescoço, tronco, coordenação motora, duas ou três peças de vestir, pernas, pés, pescoço integrado, pernas e braços na posição correta, pupilas, ombros, cabelos, braços, dedos, olhos, braço, cotovelo-ombro, contorno de braços e pernas, contorno do tronco, traços fisionômicos, desenho sem transparência, vestimenta coerente, contorno da cabeça, boca e nariz, quatro peças de vestir, queixo, orelhas. Na correção do instrumento, foi atribuído um ponto à presença de cada um dos itens anteriormente mencionados e zero à sua ausência, sendo que o escore geral foi obtido pela soma desses itens. Dessa forma, a máxima pontuação que uma criança pôde obter nesse teste foi 30 .

Essa escala apresenta 30 itens que diferenciaram todas as idades estudadas de sete a 10 anos conforme demonstrado pela prova de Tukey. Observou-se, por meio da análise de funcionamento diferencial do item a necessidade de se separar duas escalas em razão do sexo. Os critérios para a seleção dos itens em cada escala foram a saturação em $g$, a diferenciação por idade $e$ a unidimensionalidade da escala, sendo que os indicadores que atenderam a dois desses critérios permaneceram na versão final do sistema de avaliação. 0 Manual apresenta ainda uma classificação hierárquica dos itens por idade e sexo da criança que fornece uma informação a mais na interpretação dos dados (Sisto, 2005). 
Nos estudos apresentados no Manual, demonstrou-se não ser necessária uma quantidade grande de itens na análise dos desenhos da figura humana, retomando a idéia inicial do teste de Goodenough de facilidade e rapidez na aplicação. Além disso, verificou-se que não é necessário solicitar mais de um desenho, já que somente o desenho de uma pessoa humana foi suficiente para a avaliação da capacidade intelectual das crianças (Sisto, 2005). Em que pese esses dados, verificou-se ainda estudos que demonstraram a utilidade desse instrumento na avaliação da inteligência. Nesse contexto, a pesquisa de Rueda (2005) relacionou os escores do DFH-Escala Sisto com as Matrizes Progressivas Coloridas de Raven, encontrando correlações entre moderadas nas diferentes faixas etárias e por sexo. Desse modo, sugere que o DFH estaria medindo em parte o mesmo constructo que o Matrizes Progressivas Coloridas de Raven. No entanto, há que se considerar os problemas já mencionados quanto este teste. Além disso, Sisto (2000) verificou correlações das pontuações no DFH com as provas piagetianas, indicando que esse instrumento forneceria uma estimativa das capacidades inteletuais de crianças.

\section{Procedimento}

A aplicação foi coletiva e realizada em sala no período das aulas, em um horário previamente cedido pelas professoras, sendo entregue lápis, borracha e uma folha de papel sulfite A4 para que pudessem fazer a cópia das figuras do teste de Bender bem como o DFH. Foi instruído que as crianças fizessem as cópias das figuras do Bender o melhor possível, sendo que, quando terminassem de copiar, seria trocado a figura por outra e assim sucessivamente. As dúvidas quanto à execução das cópias dos desenhos foram respondidas solicitando para que fizessem o mais parecido que conseguissem. Foi solicitado que fizessem, numa página da folha, a cópia das figuras do Teste Gestáltico e no seu verso o DFH. Vale ressaltar que, nesse estudo, as figuras do Teste Gestáltico de Bender foram projetadas no quadro por um retroprojetor para que pudessem ser mais bem visualizadas por todos, facilitando, assim a aplicação coletiva desse instrumento.

\section{RESULTADOS}

A distribuição da pontuação do Bender para os sujeitos dos grupos extremos constituídos com base no escore do DFH encontra-se na Figura 1. A 
média foi 9,10 ( $\mathrm{DP}=4,37)$ indicando que a maior parte dos sujeitos obteve escores abaixo do ponto médio da escala para esse instrumento, sugerindo poucas distorções na cópia das figuras. Observou-se que 51,8\% dos participantes pontuaram entre dois e oito no Bender. Entretanto, houve crianças $(3,6 \%)$ que fizeram distorcidas a maior parte das cópias com escore entre 18 e 20 pontos.

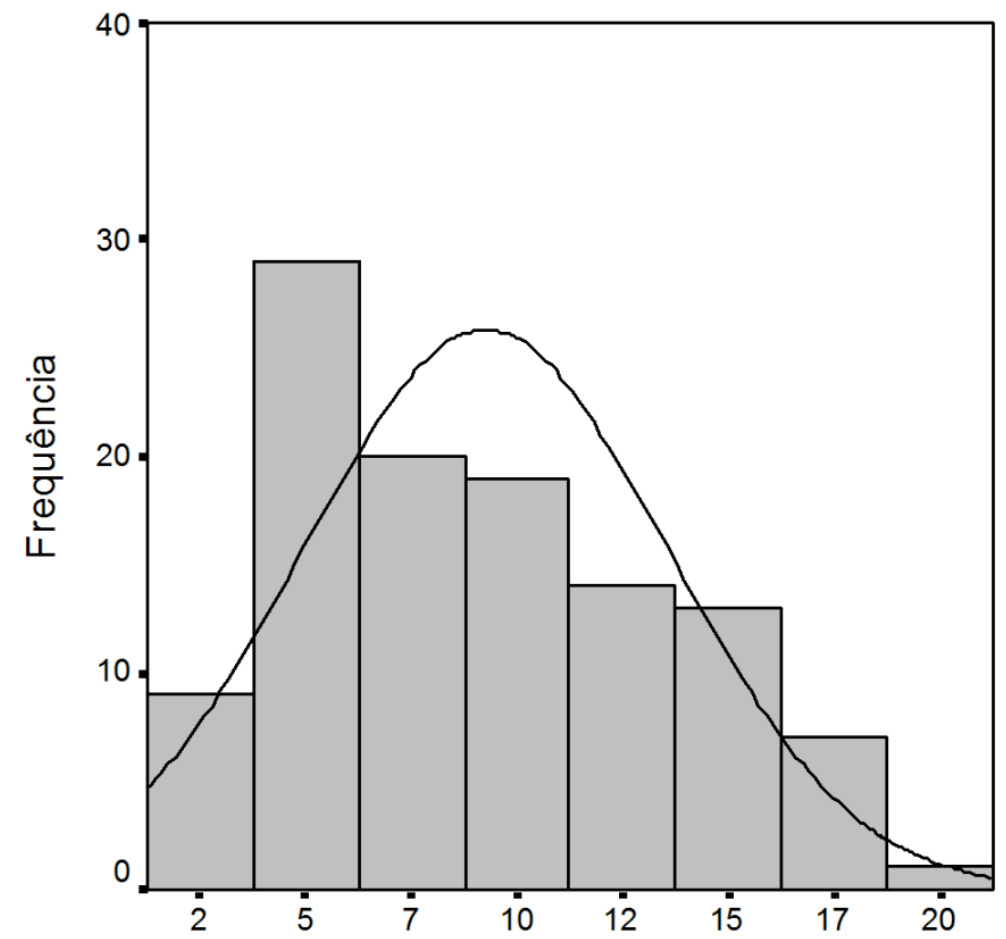

Pontuação total do Bender

Figura 1. Distribuição da Pontuação do Bender $(\mathrm{N}=112)$.

Para investigar a discriminação das medidas feitas do teste Gestáltico de Bender, foram separados grupos de pontuações extremas do DFH por idade e sexo e no geral. Para isso, foram calculados os quartis em cada idade e sexo e também com todas as idades. Os critérios para a formação dos grupos do DFH estão descrito na Tabela 1 , na qual o grupo 1 apresenta o ponto de corte referente a $25 \%$ das crianças com menores pontuações no DFH e o grupo 2 o ponto que separa $25 \%$ dos participantes com os maiores escores nesse teste. 
Tabela 1.

Níveis de corte para formação dos grupos extremos em cada idade e no geral para ambos os sexos no DFH $(\mathrm{N}=112)$.

Masculino

Grupo 1

Grupo 2

\begin{tabular}{ccc}
\hline 7 anos & Menos de 6 pontos & Mais de 11 pontos \\
8 anos & Menos de 7 pontos & Mais de 14,75 pontos \\
9 anos & Menos de 10 pontos & Mais de 19,5 pontos \\
10 anos & $\begin{array}{c}\text { Menos de } 12 \text { pontos } \\
\text { Geral }\end{array}$ & $\begin{array}{c}\text { Mais de } 22 \text { pontos } \\
\text { Menos de } 8 \text { pontos }\end{array}$ \\
\hline \multicolumn{2}{c}{ Feminino } \\
\hline 7 anos & Menos de 6,5 pontos 18 pontos \\
8 anos & Menos de 5,5 pontos & Mais de 11,5 pontos \\
9 anos & Menos de 10,5 pontos & Mais de 12 pontos 19,5 pontos \\
10 anos & Menos de 14 pontos & Mais de 20 pontos \\
Geral & Menos de 8 pontos & Mais de 18 pontos \\
\hline
\end{tabular}

A diferença entre esses grupos foi estudada por meio da prova t de Student, estabelecendo nível de significância de 5\%. Os resultados dessa análise estão na Tabela 2.

Tabela 2. Médias, desvios padrão, valores t de Student e níveis de significância (p) das pontuações do teste de Bender nos grupos extremos do DFH, por idade, sexo e geral $(\mathrm{N}=112)$.

\begin{tabular}{|c|c|c|c|c|c|c|}
\hline \multirow{2}{*}{ Idade } & \multirow[b]{2}{*}{ Sexo } & \multirow[b]{2}{*}{ Grupo } & \multicolumn{4}{|c|}{ Teste de Bender } \\
\hline & & & $M$ & $d p$ & $t$ & \multirow{2}{*}{$\frac{p}{0,000}$} \\
\hline \multirow{2}{*}{$\begin{array}{c}7 \\
\text { anos }\end{array}$} & Masculino & $\begin{array}{l}\text { Grupo } 1 \\
\text { Grupo } 2\end{array}$ & $\begin{array}{c}12,89 \\
7,53\end{array}$ & $\begin{array}{l}3,70 \\
3,22\end{array}$ & 7,86 & \\
\hline & Feminino & $\begin{array}{l}\text { Grupo } 1 \\
\text { Grupo } 2 \\
\end{array}$ & $\begin{array}{c}12,89 \\
7,22 \\
\end{array}$ & $\begin{array}{l}3,70 \\
3,03 \\
\end{array}$ & 8,64 & 0,000 \\
\hline \multirow{2}{*}{$\begin{array}{c}8 \\
\text { anos }\end{array}$} & Masculino & $\begin{array}{l}\text { Grupo } 1 \\
\text { Grupo } 2\end{array}$ & $\begin{array}{c}12,60 \\
6,85\end{array}$ & $\begin{array}{l}3,88 \\
2,76\end{array}$ & 9,95 & 0,000 \\
\hline & Feminino & $\begin{array}{l}\text { Grupo } 1 \\
\text { Grupo } 2 \\
\end{array}$ & $\begin{array}{c}12,65 \\
7,22 \\
\end{array}$ & $\begin{array}{l}4,11 \\
3,03\end{array}$ & 7,10 & 0,000 \\
\hline \multirow{2}{*}{$\begin{array}{c}9 \\
\text { anos }\end{array}$} & Masculino & $\begin{array}{l}\text { Grupo } 1 \\
\text { Grupo } 2\end{array}$ & $\begin{array}{c}11,22 \\
5,76\end{array}$ & $\begin{array}{l}4,00 \\
2,23\end{array}$ & 8,57 & 0,000 \\
\hline & Feminino & $\begin{array}{ll}\text { Grupo } 1 \\
\text { Grupo } 2 \\
\end{array}$ & $\begin{array}{c}11,22 \\
5,76 \\
\end{array}$ & $\begin{array}{l}4,00 \\
2,23 \\
\end{array}$ & 8,57 & 0,000 \\
\hline \multirow{2}{*}{$\begin{array}{c}10 \\
\text { anos }\end{array}$} & Masculino & $\begin{array}{ll}\text { Grupo } 1 \\
\text { Grupo } 2\end{array}$ & $\begin{array}{c}10,82 \\
5,78\end{array}$ & $\begin{array}{l}4,03 \\
2,31\end{array}$ & 6,26 & 0,000 \\
\hline & Feminino & $\begin{array}{l}\text { Grupo } 1 \\
\text { Grupo } 2 \\
\end{array}$ & $\begin{array}{c}10,47 \\
5,76\end{array}$ & $\begin{array}{l}4,01 \\
2,23\end{array}$ & 7,55 & 0,000 \\
\hline \multirow[t]{2}{*}{ Geral } & Masculino & $\begin{array}{l}\text { Grupo } 1 \\
\text { Grupo } 2\end{array}$ & $\begin{array}{c}11,69 \\
6,38\end{array}$ & $\begin{array}{l}3,97 \\
2,77\end{array}$ & 8,74 & 0,000 \\
\hline & Feminino & $\begin{array}{l}\text { Grupo } 1 \\
\text { Grupo } 2\end{array}$ & $\begin{array}{c}11,69 \\
6,38\end{array}$ & $\begin{array}{l}3,97 \\
2,77\end{array}$ & 8,74 & 0,000 \\
\hline
\end{tabular}


Pelos dados da Tabela 2, verifica-se que, tanto no geral por sexo, como separadamente por idade, os grupos extremos formados com base nas pontuações do DFH são diferenciados pelos escores do Teste de Bender. Em todos os casos observados, as médias do grupo 1 foram maiores que no grupo 2, indicando que as crianças com maior maturidade conforme avaliadas pelo DFH evidenciam menos distorções nas cópias do teste de Bender, e melhor maturidade visomotora. Um dado que se observou foi que as diferenças entre as idades, embora significativas, foram pequenas.

\section{DISCUSSÃO}

Este estudo teve por objetivo buscar evidências de validade entre o DFH com base nos critérios de avaliação propostos por Sisto (2005) e o B-SPG. Anastasi e Urbina (2000) ressaltaram que uma das formas de se obter esse tipo de informação seria separando grupos extremos na medida do critério e verificando se seriam diferenciados em razão das pontuações do instrumento que se quer validar. Esse tipo de procedimento foi adotado nessa pesquisa. Por um lado, valeu-se da premissa de que ambos os instrumentos teriam aspectos comuns entre si, como o fato de envolverem aspectos motores e de mensurarem variáveis que apresentam um caráter desenvolvimental (Koppitz, 1989; Baer \& Gale, 1967; Doubros \& Mascarenhas, 1969). Por outro, a literatura que se dispõe sobre o Bender e inteligência tem revelado que o Teste Gestáltico pode ser usado para se obter uma ideia da capacidade intelectual de crianças. Entretanto, com o B-SPG, em relação à inteligência, somente o trabalho de Sisto, Bartholomeu, Rueda, Santos e Noronha (2008) evidenciou correlações com o teste Matrizes Progressivas Coloridas de Raven, devendo-se levar em consideração que este instrumento não é unidimensional, o que não se podendo afirmar que avalie o fator g, tal como é proposto em seu Manual (Sisto, Rueda \& Bartholomeu, 2006). Desse modo, questionou-se o uso desse instrumento enquanto medida de critério, e, por isso, valeu-se do DFH-Escala-Sisto (Sisto, 2005) como medida de inteligência para se tentar estabelecer relações com o Bender.

De fato, ambos os instrumentos baseiam-se em produções gráficas das crianças para aferir características psicológicas. Nesse sentido, é importante salientar que a capacidade de reproduzir cópias de figuras com poucas distorções (ressaltando boa maturidade viso-motora no teste de Bender) também afetaria 
uma boa coordenação na produção de desenhos da figura humana, sendo esperada, portanto, alguma relação entre os resultados nessas duas provas que se justificaria pela habilidade gráfica da pessoa e não somente pelas associações entre maturação viso-motora e inteligência, constructos subjacentes às provas. Assim, apesar de no presente estudo esses dois testes estarem relacionados, não se tem clareza ainda de quanto dessa relação pode ser explicada pela habilidade gráfica dos sujeitos, pelas relações entre habilidade viso-motora e inteligência. Tal fato convida a novas pesquisas. Outra possibilidade de associação entre esses testes seria decorrente do fato de ambos tratarem de variáveis que são alteradas com o desenvolvimento, sendo que esse último justificaria as relações entre os dois testes. Nesta pesquisa, o controle por idade feito revela que existem associações entre esses dois testes independentemente da idade (separadamente em cada faixa etária), controlando-se esta característica.

A literatura dispõe de poucos trabalhos que relacionaram esses dois instrumentos, o que dificulta a discussão dos resultados encontrados. Todavia, dos poucos estudos encontrados na literatura que relacionaram o DFH, como medida de inteligência, e o Bender, alguns merecem destaque. É o caso da pesquisa de Yousefi, Shahim, Razavieh e Mehryar (1992) que, estudando crianças iranianas com o DFH de Goodenough-Harris e o Teste de Bender pelo sistema de Koppitz, encontraram correlações entre esses instrumentos, sugerindo que o Bender poderia ser empregado na estimativa das capacidades intelectuais de crianças. Na presente pesquisa, o procedimento de análises adotado, embora não tenha sido o mesmo, permitiu também identificar que o BSPG discriminou crianças com maiores escores no DFH das com menores escores. Também, Rueda, Bartholomeu e Sisto (2004) estudaram as relações entre esses instrumentos pelos sistemas Koppitz (no caso do Bender) e de Goodenough-Harris (para o DFH) e observaram correlações negativas e significativas entre a distorção, integração e total do Bender com o DFH. Desse modo, a maturidade viso-motora estaria relacionada à qualidade dos desenhos tal como sugeriram os autores. Essas conclusões também podem ser afirmadas na presente pesquisa, uma vez que, nesse estudo, a análise de grupos extremos formados pela pontuação total do DFH indicou diferenças significativas no escore do B-SPG em todas as situações estudadas. Desse modo, a medida do B-SPG diferenciou crianças mais inteligentes das menos inteligentes, tal como 
mensurado pelo DFH-Escala Sisto. Esses dados estão de acordo com os demais evidenciados na literatura sobre esses instrumentos.

Considerando os descritos anteriormente, a inteligência seria uma das características que as crianças com boa maturidade visomotora apresentariam e deveria contribuir com uma parte importante da variância do Bender. Nesse contexto, os dados desse estudo podem ser considerados evidência de validade concorrente por grupos contrastantes para o B-SPG em relação ao DFH-Escala Sisto. Tais achados estão de acordo com o que é esperado e mencionado na literatura, de forma geral (Rueda, Bartholomeu \& Sisto, 2004; Yousefi, Shahim, Razavieh \& Mehryar, 1992; Mcnamara, Porterfield \& Miller, 1969; Silberberg \& Feldt, 1968). Uma última consideração deve ser feita quanto aos constructos estudados, poderia se dizer que crianças com maior maturidade visomotora apresentariam também maior qualidade nos desenhos, já que, aparentemente, quanto menos distorções nas cópias das figuras do Bender esses sujeitos evidenciaram, mais detalhes tenderam a incluírem nos desenhos.

\section{CONSIDERAÇÕES FINAIS}

Segundo Bender (1938) a função gestáltica possibilita ao organismo constituir padrões a partir das respostas aos estímulos do meio, uma vez que encerra uma gestalt. Considerando os resultados encontrados nesse trabalho, sob a perspectiva desse construto teórico, pode-se referir que a execução dos desenhos de figura humana também envolveriam essa capacidade, constituindo, de certa forma, um padrão igualmente. Todavia, os dados encontrados não possibilitaram averiguar em que medida a habilidade artística dos sujeitos estaria imbricada na qualidade dos desenhos. Desse modo, poderia ser verificado quais outras variáveis estariam imbricadas nas relações entre esses instrumentos tais como a própria habilidade artística ou criatividade tal como é sugerido na literatura (Aikman, Belter \& Finch, 1992). Isso requer pensar em quais outros aspectos estariam envolvidos na execução das cópias das figuras; em outros termos, em quais outros mecanismos psicológicos estariam envolvidos na relação existente entre o Teste de Bender e o DFH. Além disso, essas relações foram estabelecidas somente em crianças de escolas públicas, de tal forma que não permitem inferir os resultados para alunos de escolas particulares, já que apresentam características sócioeconômicas diferentes que poderiam talvez 
alterar em algumas partes os resultados ora obtidos, podendo-se investigar essas diferenças em crianças com essas características.

Por fim, no âmbito das evidências de validade, esses resultados encontrados podem ser considerados como evidência por grupos contrastantes para os instrumentos em questão. No entanto, há a necessidade de que novas pesquisas sejam conduzidas para investigar esse tipo de informação para o BSPG em relação à inteligência com outros critérios diferentes do DFH, já que, concordando com Anastasi e Urbina (2000), há a necessidade do acúmulo de evidências de validade dos instrumentos de avaliação psicológica, no sentido de se complementarem e fornecerem, cada vez mais, uma compreensão mais ampla dos escores dos testes em diferentes situações, para que estes forneçam avaliações mais adequadas aos clientes dos psicólogos.

\section{REFERÊNCIAS}

Aikman, K. G.; Belter, R. W. \& Finch, A. J. (1992). Human Figure Drawings: validity in assessing intellectual level and academic achievement. Journal of Clinical Psychology, 48(1), 114-120.

Anastasi, A. \& Urbina, S. (2000). Testagem Psicológica. Artes Médicas: Porto Alegre.

Baer, D. J. \& Gale, R. A. (1967). Intelligence and Bender Gestalt test performance of institutional and noninstitutional school children. Journal of Genetic Psychology, 111(1) 119-124.

Baldwin, M. V. (1950). A note regarding the suggested use of the Bender Gestalt Test as a measure of school readiness. Journal of Clinical Psychology, 6, 142-155.

Bartholomeu, D., Rueda, F. J. M. \& Sisto, F. F. (2006). Teste de Bender e dificuldades de aprendizagem: Evidência de validade. Avaliação Psicológica, $4(1), 13-22$.

Bender, L. (1938). A visual motor Gestalt test and its clinical use. The american Orthopsychiatric Association: New York.

Billingslea, F. (1948). The Bender-Gestalt: An objective scoring method and validating data. Journal of Clinical Psychology, 4, 1-27.

Cronbach, J. E. (1996). Fundamentos da Testagem Psicológica. Porto Alegre: Artes Médicas. 
Doubros, S. G. \& Mascarenhas, J. (1969). Relations among Wechsler full-scale scores, organicity sensitive subtest scores and Bender Gestalt Errors scores. Perceptual and Motor Skills, 29(3) 719-722.

Gobetz, W. (1953). A quantification, standardization and validation of the Bender-Gestalt Test on normal and neurotic adults. Psycho Mono, 67(6) 356-360.

Harriman, M. \& Harriman, P. (1950). The Bender-Gestalt as a measure of school readiness. Journal of Clinical Psychology, 6, 175-177.

Hilgert, L. D. \& Adams, W. F. (1989). Using the Bender Gestalt Test to predict graphomotor dimensions of the Draw a person test. Perceptual and Motor Skills, 68(1), 27-32.

Koppitz, E. M. (1989). O teste gestáltico Bender para crianças. Trad.: Rosaura Nemoto Piccoli, Porto Alegre: Artes Médicas.

Kroeff, P. (1988). Normas brasileiras para o Teste de Bender. Psicologia: Reflexão e Crítica, 1/2(3), 12-19.

Kroeff, P. (1992). Desempenho de crianças no Teste de Bender e nível sócioeconômico-cultutal. Psicologia: Reflexão e Crítica, 5(2), 119-126.

Mcnamara, J. R., Porterfield, C. L. \& Miller, L. E. (1969). The relationship of the Wechsler Preschool and Primary Scale of Intelligence with the Coloured Progressive Matrices and the Bender Gestalt Test. Journal of Clinical Psychology, 25(1), 65-68.

Pinelli Jr., B. (1990). Adaptação do "Teste do Desenvolvimento da Integração Viso-Motora (VMI)" para uso no Brasil. Dissertação de Mestrado, Universidade de Brasília, Brasília/DF, 184p.

Rueda, F. J. M. (2005). DFH-Escala Sisto e Matrizes Progressivas Coloridas de Raven: Evidências de validade. Dissertação de Mestrado, Programa de PósGraduação Stricto Sensu em Psicologia, Universidade São Francisco, Itatiba. Rueda, F. R. M., Bartholomeu, D. \& Sisto, F. F. (2004). Desenho da figura humana e aprendizagem da escrita. In: C. Machado, L. Almeida, M. Gonçalves \& V. Ramalho. Avaliação Psicológica: Formas e Contextos. Psiquilíbrios Edições. Braga. p. 236-243.

Silberberg, N. \& Feldt, L. S. (1968). Intellectual and perceptual correlates of reading difficulties. Journal of School Psychology, 6(4), 237-245.

Sisto, F. F. (2000). Relationship of the Piagetian cognitive development to human figure drawing. Child Study Journal, 30(4), 225-232. 
Sisto, F. F. (2005). DFH-Escala Sisto. São Paulo: Vetor Editora.

Sisto, F. F., Noronha, A. P. P. \& Santos, A. A. A. (2004). Distorção de forma no Teste de Bender: Questionando seu critério de validade. Revista do Departamento de Psicologia da UFF, 16(2), 139-154.

Sisto, F. F., Noronha, A. P. P. \& Santos, A. A. A. (2005). Bender - Sistema de Pontuação Gradual B-SPG. Vetor Editora: São Paulo.

Sisto, F. F., Rueda, F. J. M. \& Bartholomeu, D. (2006). Estudo sobre a unidimensionalidade do Teste Matrizes Progressivas Coloridas de Raven. Psicologia: Reflexão e Crítica, 3, 111-121.

Sisto, F. F., Santos, A. A. A. \& Noronha, A. P. P. (2006). Critério de integração do Teste de Bender: explorando evidências de validade. Avaliação Psicológica, $3(1), 17-32$.

Suehiro, A. C. B. (2005). O Bender e as dificuldades de aprendizagem: evidências de validade. Dissertação de Mestrado, Programa de PósGraduação Stricto Sensu em Psicologia, Universidade São Francisco, Itatiba, $110 p$.

Sundberg, N. D. (1961). The practice of psychological testing in clinical services in the United States. American Psychologist, 16, 19-43.

Vance, B., Fuller, G. B. Lester, M. L. (1986). A comparison of the Minnesota Perceptual Diagnostic test revised and the Bender Gestalt. Journal of Learning Disabilities, 19(4), 211-214.

Yousefi, F., Shahim, S., Razavieh, A. \& Mehryar, A. H. (1992). Some normative data on the Bender Gestalt Test performance of Iranian children. British Journal of Educational Psychology, 62(3), 410-416.

Sisto, F. F., Bartholomeu, D., Rueda, F. J. M. Santos, A. A. A. \& Noronha, A. P. P. (2008). Relações entre os testes de Bender e Matrizes progressivas coloridas de Raven na avaliação da inteligência. Interação em Psicologia, 12(1), 11-19.

Contato: d_bartholomeu@yahoo.com.br

Recebido em: 12/12/2012

Revisado em: 20/12/2012

Aceito em: 29/12/2012 